\title{
CONTROLE SEMI-ATIVO APLICADO A VIBRAÇÕES EXCESSIVAS DE UMA SUSPENSÃO AUTOMOTIVA
}

\section{SEMI-ACTIVE CONTROL APPLIED TO EXCESSIVE VIBRATIONS OF AN AUTOMOTIVE SUSPENSION}

\author{
Luiz Gustavo Pereira Roéfero ${ }^{1}$; Fábio Roberto Chavarette ${ }^{2}$ \\ ${ }^{1}$ Universidade Estadual Paulista - UNESP, Departamento de Engenharia Mecânica \\ E-mail: Igpr98gu@gmail.com \\ ${ }^{2}$ Universidade Estadual Paulista - UNESP, Departamento de Matemática \\ E-mail: fabio.chavarette@unesp.br
}

RESUMO - Automóveis são sistemas bem presentes no cotidiano da população, seja para o uso no trabalho ou em atividades de lazer. Dentre os diversos equipamentos presentes em um automóvel, a suspensão é qualificada como uma das mais importantes pois, além de manter a integridade física da estrutura, auxilia na confortabilidade dos passageiros. Neste sentido o dimensionamento desta suspensão e o efeito de amortecimento desempenhado pela mesma deve ser minuciosamente estudado, pois qualquer erro pode acarretar no aparecimento de falhas e acabar levando à perda financeira ou até colocar em risco vidas humanas. Com isso, achou-se interessante o estudo da dinâmica de um Amortecedor Magneto-Reológico em um modelo de suspensão automotiva já proposto na literatura, visando a melhoria de desempenho dinâmico do modelo. Para efetuar esta análise, utilizou-se o método de Runge Kutta de Quarta Ordem visando a aproximação numérica tanto do sistema sem o controle empregado, quanto do modelo já controlado.

Palavras-chave: Magneto-Reológico; Suspensão Automotiva; Dinâmica.

ABSTRACT - Automobiles are well present in the daily lives of the population, whether for use at work or in leisure activities. Among the various equipment present in a car, the suspension is qualified as one of the most important because, in addition to maintaining the physical integrity of the structure, it assists in the comfort of the passengers. In this sense, the dimensioning of this suspension and the damping effect performed by it must be thoroughly studied, as any error can lead to the appearance of failures and end up leading to financial loss or even endanger human lives. Thus, it was interesting to study the dynamics of a magneto-rheological damper in an automotive suspension model already proposed in the literature, aiming to improve the dynamic performance of the model. In order to carry out this analysis, the Runge Kutta method of the Fourth Order was used aiming at the numerical

Recebido em: 21/08/2018 Revisado em: 04/09/2018 Aprovado em: 10/09/2018 approximation of both the non-damping system and the already damped model.

Keywords: Magneto-Rheological; Automotive Suspension; Dynamics. 


\section{INTRODUÇÃO}

Em 1886 surgiram os primeiros automóveis movidos à gasolina, cujo mérito é dado a Karl Benz (1844-1929) e Gottlieb Daimler (1834-1900). Já em 1908, a indústria automobilística estava muito bem instalada nos Estados Unidos com a presença de Henry Ford e a fundação da General Motors Corporation (PICADO, 1998).

Dentro deste conceito de um sistema automobilístico, é necessário que a integridade estrutural do dispositivo projetado seja mantida durante o maior tempo possível de operação. Neste sentido, é comum de se encontrar em automóveis, diversos tipos de filtros de vibração que protegem a estrutura física tanto da excitação promovida pelo motor de combustão interna quanto de estímulos dados pelo meio, como por exemplo uma irregularidade no terreno a ser percorrido (SÁNCHEZ, 2013).

Sem dúvida alguma, o filtro de vibração com maior grau de importância dentro do projeto de um automóvel é a Suspensão, que atua impedindo que qualquer estímulo externo que seja fornecido ao pneu atinja de forma brusca o restante da estrutura. Caso não houvesse tal mecanismo, todo o projeto estaria exposto à muitos danos causados por terrenos bastante irregulares.

Dentre os tipos de amortecedores presentes em suspensões automobilística, vem sendo estudado a bastante tempo os Amortecedores Magneto-Reológicos que tem como característica a capacidade de alteração do seu coeficiente de amortecimento de acordo com o campo magnético gerado por uma corrente que é fornecida ao sistema (TUSSET et al, 2012). Quanto maior a corrente, maior será seu coeficiente de amortecimento (TUSSET E BALTHAZAR, 2012).

Outra característica que torna o Amortecedor Magneto-Reológico usual, é a sua capacidade de controle de sistemas (CUNHA et al, 2013). Esta habilidade faz com que o a estrutura na qual ele está aplicado não desempenhe funções instáveis ou caóticas (PRADO e FIEDLER-FERRARA, 1994).

Como qualquer sistema dinâmico, é possível que este dispositivo desempenhe seu comportamento fora do previsto caso não seja dimensionado de forma correta (MONTEIRO, 2012). Devido a este fato, é muito viável que se modele matematicamente o projeto e realize-se uma simulação numérica visando a predição de comportamento deste sistema (SAVI, 2006).

Neste trabalho buscou-se aperfeiçoar - modelo utilizado por Sánchez (2013) utilizando um Amortecedor MagnetoReológico, visando obter uma suspensão automotiva com melhor desempenho que a apresentada na literatura citada anteriormente (SÁNCHEZ, 2013).

\section{METODOLOGIA}

Para a realização deste trabalho foi utilizado um modelo de Amortecedor Magneto-Reológico muito recorrente na literatura. Este modelo foi estudado no trabalho elaborado por Tusset e Balthazar (2012).

\subsection{Amortecedor Magneto-Reológico}

Existem diversos modelos matemáticos que conseguem simular o comportamento dinâmico de um amortecedor deste tipo, entretanto o mais recorrente na literatura é o modelo de BoucWen (TUSSET e BALTHAZAR, 2012) cujo modelo físico é ilustrado na figura 1. 
Figura 1. Modelo de Bouc-Wen.

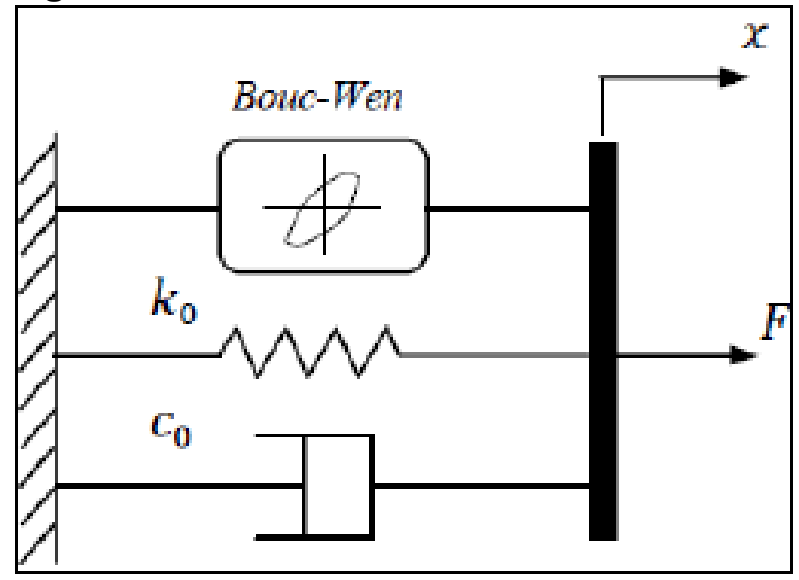

Fonte: (TUSSET e BALTHAZAR, 2012).

Este amortecedor desempenha uma força $F$ que é calculada pela equação (1).

$F=c_{0} \dot{x}+k_{0}\left(x-x_{0}\right)+\alpha z$

(1)

Em que a variável $z$ é calculada pela equação 2.

$$
\dot{z}=-\lambda|\dot{x}| z|z|^{n-1}-\gamma \dot{x}|z|^{n}+\beta \dot{x}
$$

Nas equações (1) e (2), c c e ko são, em sequência, o coeficiente de amortecimento viscoso e de rigidez elástica, $x_{0}$ é 0 deslocamento inicial, $x$ é a variável dependente, a é um coeficiente de rigidez e as constantes $\lambda, \gamma, \beta$ e $n$ dependem das características do amortecedor. Ajustando-se os parâmetros do modelo (em especial $\lambda, \gamma$ e $\beta)$, pode-se controlar a linearidade no descarregamento e a suavidade da transição na inversão da velocidade (regiões de précarga e pós-carga). Além disso, a força do acumulador, $f_{0}$, pode ser diretamente incorporada neste modelo através de um deslocamento inicial $x_{0}$ na mola linear $\mathrm{k}_{0}$.

Embora a rigidez do amortecedor seja controlada pela corrente que gera o campo magnético, não podemos ver ela explicitamente nas equações. Para facilitar 0 manuseio desta equação, Tusset e Balthazar (2012) reescreveram a equação que demonstra a força do Amortecedor MagnetoReológico da seguinte forma.
$F=\frac{3.2}{\left(3 e^{-3.4 i}\right)+1} \dot{x}+k_{0} x+\frac{8.5}{\left(1.28 e^{-3.9 i}\right)+1} Z$

\subsection{Modelo Estudado}

O objeto de estudo deste trabalho foi adaptado do modelo estudado no trabalho de Sánchez (2013) e o sistema físico já adaptado é mostrado na figura 2. Ele apresenta apenas uma suspensão do carro, sendo que no mesmo normalmente há quatro.

Nesta figura, $m_{s}$ simula a massa de um quarto do automóvel e $m_{r}$ simula a massa de um pneu. Cada parte com seu respectivo deslocamento na vertical.

As equações já no espaço de estados são mostradas posteriormente à figura e, decidiu-se que a variável $z_{r}$, que denota o deslocamento da excitação do sistema, fosse igual a zero. Com isso, a excitação da estrutura seria dada por uma condição inicial diferente de zero na dinâmica de $z_{r}$. Fazendo isso, considera-se que o sistema tivesse acabado de subir sobre algum obstáculo e a suspensão deve executar o amortecimento para a proteção da estrutura.

Figura 2. Modelo físico estudado.

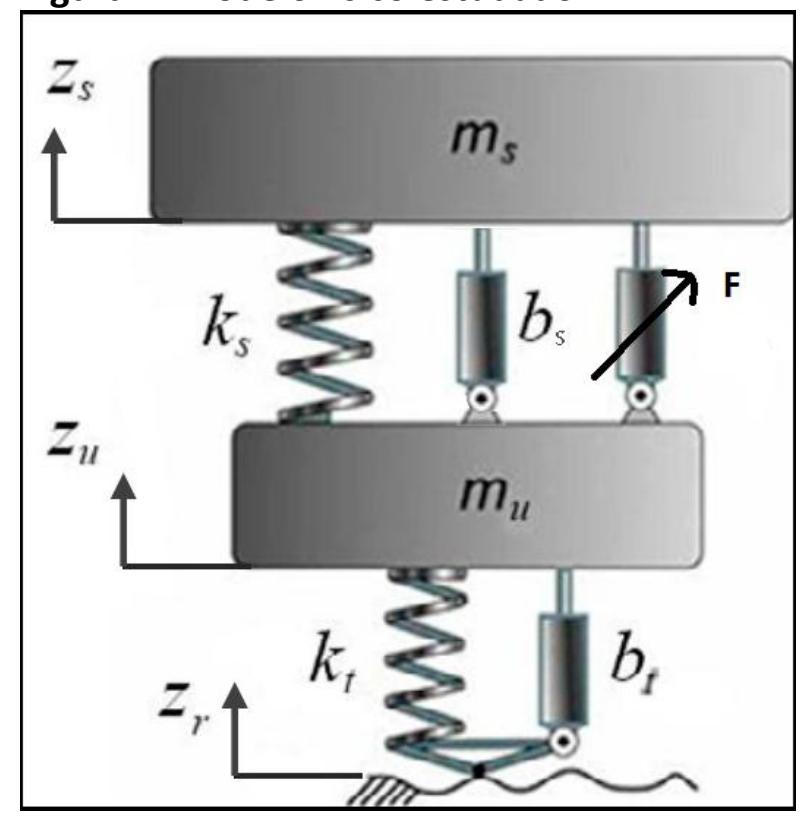

Fonte: (Tusset e Balthazar, 2012). 

seguinte.

O equacionamento deste modelo é o

$\dot{z}_{s}=y_{s}$

$\dot{y}_{s}=\frac{-b_{s}\left(\dot{z_{s}}-\dot{z_{u}}\right)-k_{s}\left(z_{s}-z_{u}\right)-F}{m_{s}}$

(5)

$$
\begin{aligned}
& \dot{z_{u}}=y_{u} \\
& \dot{y_{u}}=\frac{b_{s}\left(\dot{z}_{s}-\dot{z_{u}}\right)+k_{s}\left(z_{s}-z_{u}\right)+b_{t}\left(\dot{z_{r}-z_{u}}\right)}{m_{u}}+ \\
& \quad \frac{k_{t}\left(z_{r}-z_{u}\right)+F}{m_{u}} \\
& \dot{z_{r}}=y_{r} \\
& \dot{y_{r}}=0 \\
& \dot{z}=-\lambda|\dot{x}| z|z|^{n-1}-\gamma \dot{x}|z|^{n}+\beta \dot{x}
\end{aligned}
$$

Para executar a análise, utilizou-se o método de aproximação numérica de Runge Kutta de Quarta Ordem implementado no software livre OCTAVE (RUGGIERO e LOPES, 1996). Com este método buscou-se gráficos que mostrassem o comportamento dinâmico de cada parte do sistema.

\section{RESULTADOS}

Para realizar as simulações, optou-se por usar os parâmetros utilizados por Sánchez (2013) para simular a suspensão. Já para o Amortecedor Magneto-Reológico, decidiu-se utilizar os parâmetros apresentados por Tusset e Balthazar (2012).

Quadro 1. Parâmetros do modelo.

\begin{tabular}{|c|c|}
\hline Parâmetro & Valor \\
\hline$m_{s}$ & $208 \mathrm{~kg}$ \\
\hline$m_{u}$ & $28 \mathrm{~kg}$ \\
\hline$k_{s}$ & $18 \mathrm{~N} / \mathrm{m}$ \\
\hline$b_{s}$ & $1 \mathrm{~N} \cdot \mathrm{s} / \mathrm{m}$ \\
\hline$k_{t}$ & $127 \mathrm{~N} / \mathrm{m}$ \\
\hline$b_{t}$ & $10 \mathrm{~N} \cdot \mathrm{s} / \mathrm{m}$ \\
\hline$i$ & $1 \mathrm{~A}$ \\
\hline$k_{0}$ & $0 \mathrm{~N} / \mathrm{m}$ \\
\hline$\lambda$ & 0.1 \\
\hline$n$ & 2 \\
\hline$\beta$ & 180 \\
\hline$\gamma$ & 0 \\
\hline
\end{tabular}

Fonte: (Sánchez, 2013) e (Tusset e Balthazar, 2012).
As condições iniciais as quais o sistema foi submetido encontram-se no Quadro 2.

Quadro 2. Condições iniciais.

\begin{tabular}{|c|c|}
\hline Parâmetro & Valor \\
\hline$z_{s}$ & $0 \mathrm{~cm}$ \\
\hline$\dot{z}_{s}$ & $0 \mathrm{~cm}$ \\
\hline$z_{u}$ & $0 \mathrm{~cm}$ \\
\hline$\dot{z}_{u}$ & $0 \mathrm{~cm}$ \\
\hline$z_{r}$ & $10 \mathrm{~cm}$ \\
\hline$\dot{z_{r}}$ & $0 \mathrm{~cm}$ \\
\hline$z$ & $0 \mathrm{~cm}$ \\
\hline
\end{tabular}

Fonte: Elaborado pelo autor.

\subsection{Dinâmica sem o Amortecedor Magneto- Reológico}

Foram obtidos gráficos que mostram como é o comportamento da suspensão sem a presença do Amortecedor Magneto Reológico.

A figura 3 mostra a evolução temporal do deslocamento vertical da roda após sofrer excitação externa.

Figura 3. Deslocamento vertical da roda.

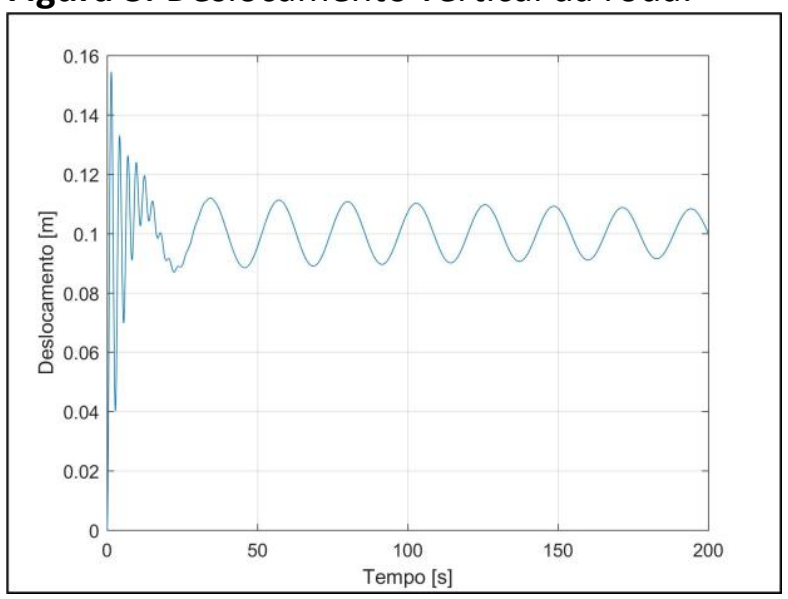

Fonte: Elaborado pelo autor.

A figura 4 apresenta a evolução da velocidade de oscilação da roda do automóvel conforme o tempo. 
Figura 4. Velocidade vertical da roda.

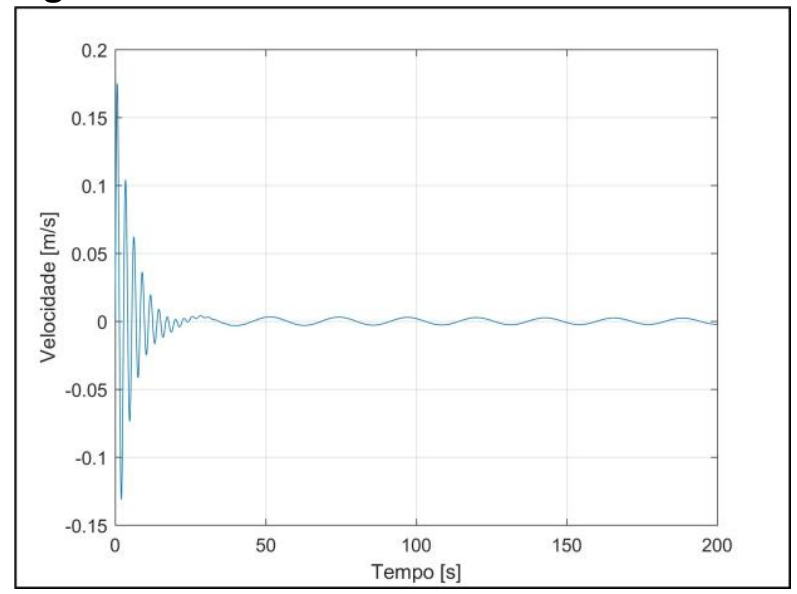

Fonte: Elaborado pelo autor.

Obteve-se também o deslocamento vertical provocado no automóvel, representado pela massa $m_{s}$.

Figura 5. Deslocamento vertical do automóvel.

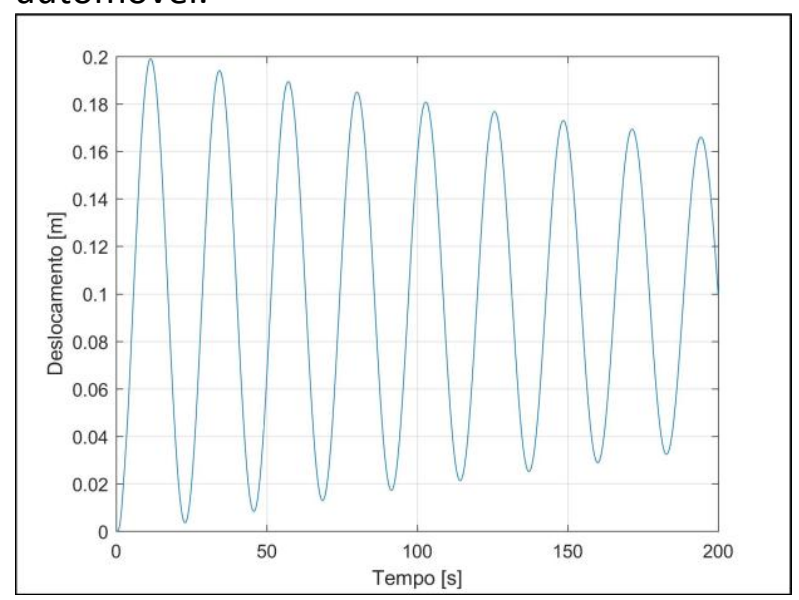

Fonte: Elaborado pelo autor.

A evolução temporal da variável $\dot{z}_{r}$, que denota a dinâmica da velocidade vertical do automóvel é mostrada na figura 6 .
Figura 6. Velocidade vertical do automóvel.

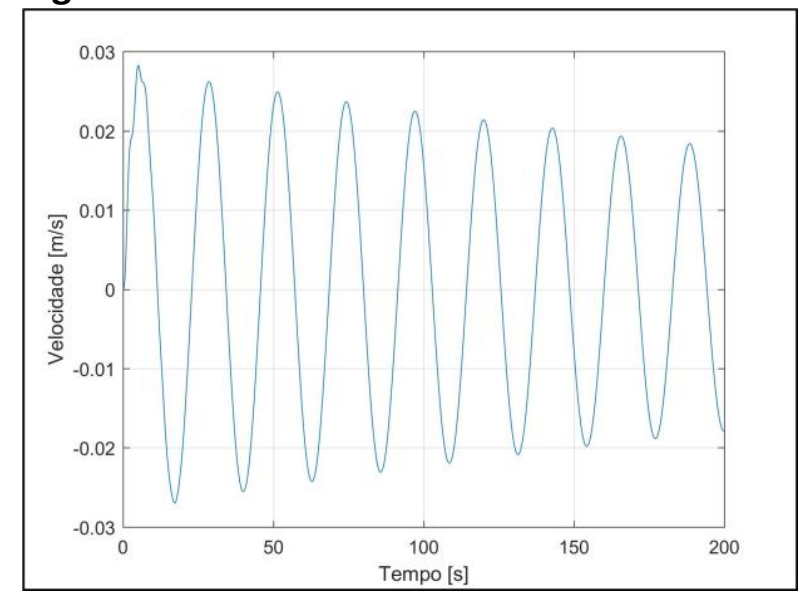

Fonte: Elaborado pelo autor.

\subsection{Dinâmica com o Amortecedor Magneto-} Reológico

Assim como na seção 3.1, foram obtidos gráficos que mostram como é o comportamento da suspensão, contudo, com a presença do Amortecedor Magneto Reológico.

A figura 7 mostra a evolução temporal do deslocamento vertical do pneu após sofrer excitação externa.

Figura 7. Deslocamento vertical da roda.

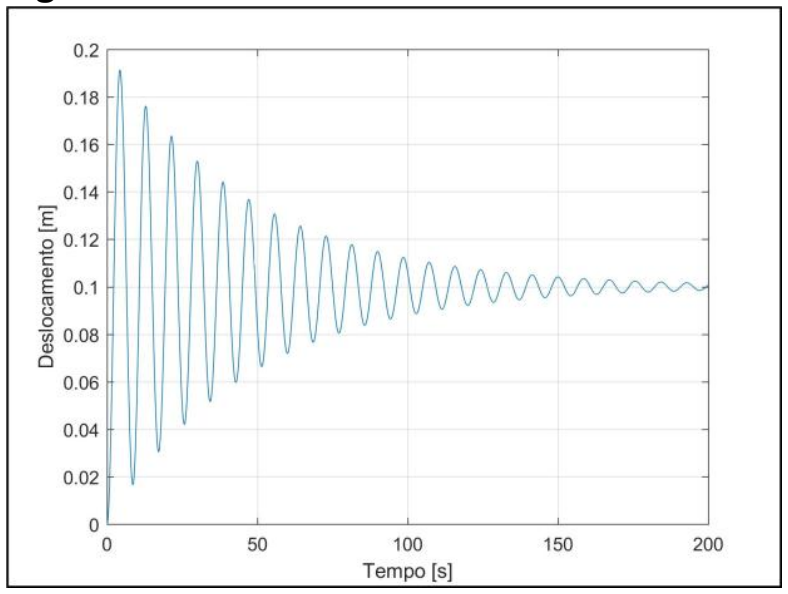

Fonte: Elaborado pelo autor.

Para fins de análise, decidiu-se observar a variação de velocidade vertical do pneu. 
Figura 8. Velocidade vertical da roda.

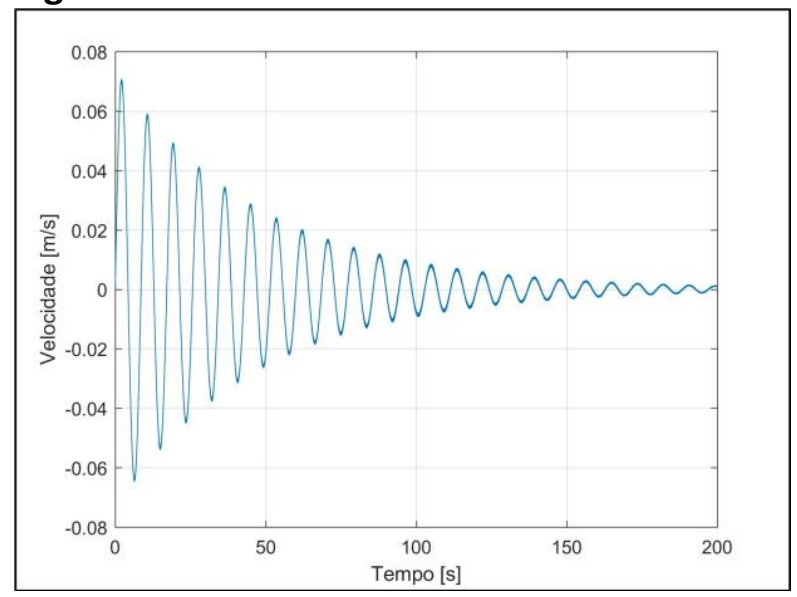

Fonte: Elaborado pelo autor.

Para analisar a o comportamento do carro após ser submetido à essa oscilação plotou-se o deslocamento vertical do automóvel.

Figura 9. Deslocamento vertical do automóvel.

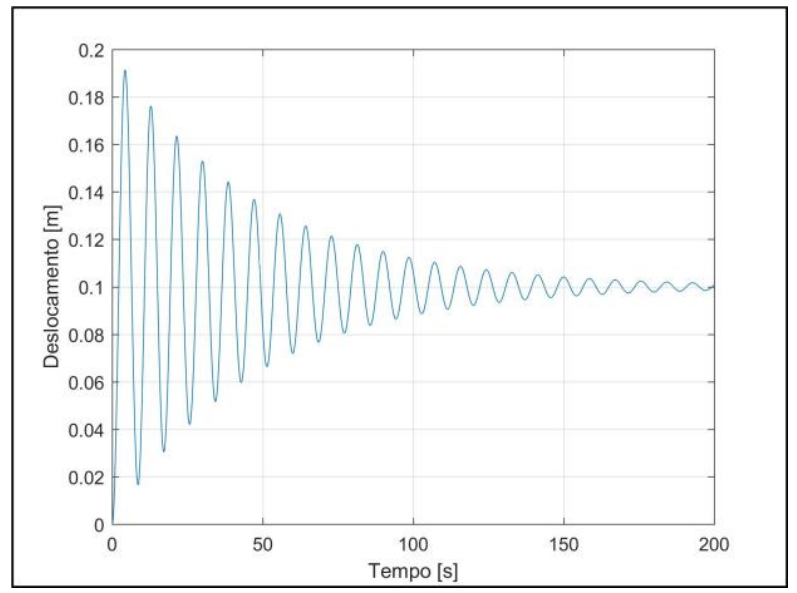

Fonte: Elaborado pelo autor.

Visando avaliação da dinâmica do automóvel, decidiu-se plotar a evolução temporal da velocidade vertical de oscilação do automóvel.
Figura 10. Velocidade vertical do automóvel.

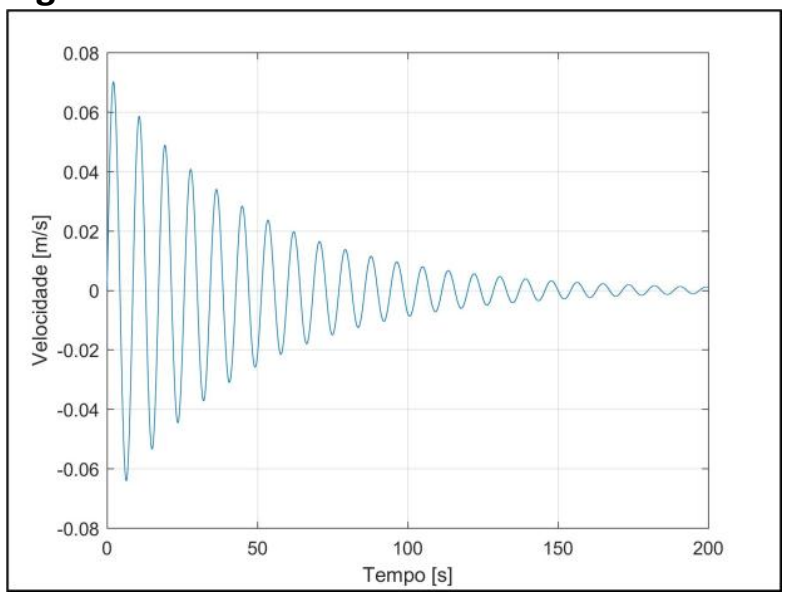

Fonte: Elaborado pelo autor.

\section{DISCUSSÕES}

Com base nos gráficos apresentados desde a figura 3 até a figura 10, todos presentes na seção 3 , podemos realizar algumas afirmações pertinentes e que diferem, de forma significativa, o modelo de suspensão sem o Amortecedor MagnetoReológico e com a presença do mesmo.

A primeira afirmação a ser feita, com base nas figuras 3 e 7 é que, sem a presença do amortecedor supracitado o sistema dissipa uma certa quantidade de energia muito rapidamente, contudo o mesmo sistema tem dificuldade em realizar o restante do amortecimento. Este comportamento faz com que oscilação fique com praticamente a mesma amplitude durante a maioria da simulação. Já com a presença do controlador, o sistema dissipa a energia da excitação de forma contínua durante todo o tempo de simulação.

Outra afirmação a ser feita é relacionada aos gráficos 5 e 9 , pois sem a presença do Amortecedor MagnetoReológico a dissipação de energia da a massa que simula o carro é bastante lenta, podendo comprometer a integridade estrutural do sistema.

As figuras 4, 6, 8 e 10 são referentes as velocidades de cada parte do modelo e foram introduzidas neste trabalho de modo a mostrar que, sem o Amortecedor MagnetoReológico, a estrutura não consegue dissipar toda a energia fornecida pela excitação. 


\section{CONSIDERAÇÕES FINAIS}

Neste trabalho verificou-se que a presença do Amortecedor MagnetoReológico acoplado ao modelo inicial auxilia na dissipação de energia fornecida pela excitação mensurada. Este auxílio faz com que o modelo estudado neste trabalho seja mais eficiente quando comparado com o modelo proposto na literatura por Sánchez (2013).

Contudo, pode-se ver que ainda sim a dissipação é considerada lenta quando analisada do ponto de vista de um sistema real. Como projeto futuro, propõe-se a melhoria deste modelo, visando uma taxa de amortecimento maior que a obtida neste trabalho e a modificação da técnica de controle para um controlador hibrido.

\section{AGRADECIMENTOS}

Os autores agradecem ao laboratório SISPLEXOS e a UNESP por oferecer sua estrutura como suporte acadêmico à pesquisa, bem como à Fundação de Amparo à Pesquisa do Estado de São Paulo (FAPESP), processo № 2017/17601-8 pelo apoio financeiro ao trabalho.

\section{REFERÊNCIAS}

CUNHA, B. S. C.; CHAVARETTE, F. R. Vibration Control Applied in a Semi-Active Suspension Using Magneto Rheological Damper and Optimal Linear Control Design. Applied Mechanics and Materials, v. 464, p. 229-234, 2013.

https://doi.org/10.4028/www.scientific.net/

AMM.464.229

GNU Octave 4.4.0 Released, 2018.

MONTEIRO, L. H. A. Sistemas dinâmicos. São Paulo: Livraria da Física, 2011. 670p.

PICADO, R. M. Controle Semi-Ativo em Suspensões Automotivas. 1998. Dissertação (Mestrado) - Universidade Estadual de
Campinas, Campinas. 1998. Disponível em: < http://repositorio.unicamp.br/bitstream/REP OSIP/264369/1/Picado_RicardoMigueis_M.p df>. Acesso em: 01 ago 2018.

PRADO, C.P.C.; FIEDLER-FERRARA, N. Caosuma introdução. São Paulo: Edgard 49 Blucher, 1994.

RUGGIERO, M. A. G.; LOPES, V. L. R. Cálculo numérico: aspectos teóricos $\mathrm{e}$ computacionais. 2. ed. São Paulo: Pearson Prentice Hall, 1996.

SÁNCHEZ, E. A. A Quarter-car Suspension System: Car Body Mass Estimator and Sliding Mode Control In: IBEROAMERICAN CONFERENCE ON ELECTRONICS ENGINEERING AND COMPUTER SCIENCE, 1., 2013, Rio de Janeiro. Anais [...] Xalapa: Elsevier, 2013. p. 208-214.

SAVI, M. Dinâmica Não-Linear e Caos. [S.I.]: Ee-papers, 2006.

TUSSET, A. M. ; BALTHAZAR, J.M. On the Chaotic Suppression of Both Ideal and Nonideal Duffing Based Vibrating Systems, Using a Magnetorheological Damper. Scientific Research and Technological Innovation, v. 21, p. 105-121, 2012.

TUSSET, A. M.; BALTHAZAR, J. M.;

CHAVARETTE, F. R.; FELIX, J. L. P. On Energy transfer phenomena, in a nonlinear ideal and non-ideal essential vibrating systems, coupled to a (MR) Magneto-rheological damper. Nonlinear Dynamics, v. 69, p. 18591880, 2012. https://doi.org/10.1007/s11071-012-0391-5 